\title{
CHROMOSCOPE: ATRIBUIÇÃO DE SENTIDOS A UM MODELO DE DISTRIBUIÇÃO ESPACIAL DE COR
}

\author{
BORDA, Adriane'; BRUM, Valentina Toaldo2; MARTINS, Gabrie/3; \\ VITALINO, Thamara Brugnhago ${ }^{\text {; }}$ GUEDES, Thiago Costa ${ }^{5}$. \\ 1 Universidade Federal de Pelotas, adribord@hotmail.com \\ 2 Universidade Federal de Pelotas, valentinatbrum@hotmail.com \\ 3 Universidade Federal de Pelotas,gabriel_m_silva@hotmail.com \\ 4 Universidade Federal de Pelotas, tbrugnhagovitalino@gmail.com \\ 5 Universidade Federal de Pelotas, cstguedes@gmail.com
}

\begin{abstract}
Resumo
Representações captam parte das coisas, subtraem, reduzem ou abstraem aspectos. O dispositivo Chromoscope resulta de um exercício de representação com o propósito de compreender e interpretar a lógica de um modelo de distribuição espacial de cor luz, o color cube, utilizado para caracterizar o universo visual digital. Objetiva provocar uma reflexão sobre como atribuir significado a este modelo para pessoas com deficiência visual. Apoiando-se na teoria da escalada da abstração de Flusser, as representações exercitadas transitam entre 0 tri e o nulodimensional. Idealizou-se um cubo que internamente se configura como um caleidoscópio, ilustrando a exigência do sentido da visão para perceber o fenômeno da cor-luz. Externamente, de maneira perceptível ao tato, representa-se uma lógica matemática relativa ao modelo em questão. Este exercício, que envolveu o uso de técnicas de desenho paramétrico e fabricação digital, criou uma narrativa que enfatiza aspectos inerentes aos sentidos da visão e do tato, ao trânsito entre modelos físicos, tridimensionais e abstratos, e, fundamentalmente, disponibilizou um recurso didático sobre os conceitos e as técnicas envolvidas.
\end{abstract}

Palavras-chave: Desenho Paramétrico; Universo Digital; Sistema RGB; Color Cube.

\begin{abstract}
Representations capture part of things, subtract, reduce or abstract aspects. The Chromoscope device results from a representation exercise with the purpose of understanding and interpreting the logic of a spatial distribution model of light color, the Color Cube, used to characterize the digital visual universe. It aims to provoke a reflection on how to assign meaning to this model for people with visual deficiency. Relying on theory of climbing of the abstraction of Flusser, the representations transit
\end{abstract}




\begin{abstract}
between three and null dimensional. It was idealized a cube that internally is configured as a kaleidoscope, illustrating the requirement of vision sense to perceive the color-light phenomenon. Externally, in a perceptible way to the touch, a mathematical logic is represented relative to the model in question. This exercise, which involved the use of parametric design and digital fabrication techniques, created a narrative that emphasizes inherent aspects of vision and touch senses, to the transit between physical, threedimensional and abstract models, and, fundamentally, provided a didactic resource about the involved concepts and techniques.
\end{abstract}

Keywords: Parametric Design; Digital Universe; RGB System; Color Cube.

\title{
1 Introdução
}

A configuração do dispositivo Chromoscope refere-se ao resultado de uma reflexão sobre como descrever a lógica de um modelo de representação de cor por meio de uma linguagem tátil. Esta reflexão foi impulsionada pelo propósito de trazer a essência da representação gráfica digital que está dirigida ao sentido visual e contrapor as experiências comunicacionais as quais transitam entre o contato direto com as coisas na sua tridimensionalidade e o contato mediado por representações. Este dispositivo, foi desenvolvido para a Expo SiGraDi 3D/20, a qual celebrou a vigésima edição do congresso anual da Sociedade Iberoamericana de Gráfica Digital durante o XX Congresso SiGraDi, em novembro de 2016, na cidade de Buenos Aires. As obras desta exposição, representando as vinte edições do congresso, foram produzidas por grupos de arquitetos, designers e artistas iberoamericanos a partir da reinterpretação e ressignificação de seus temas. O caso aqui relatado foi impulsionado pela produção do SIGraDi 2004, o qual apresentou como tema "sentido e universo digital".

O Chromoscope foi produto do significado que o tema proposto tem adquirido junto ao laboratório GEGRADI (Grupo de Estudos para Ensino/Aprendizagem de Gráfica Digital), da Universidade Federal de Pelotas, local em que foi produzido este dispositivo. Neste contexto, a abordagem tem sido direcionada à produção de modelos físicos para a comunicação tátil por meio de fabricação digital. Desta maneira tem-se tratado do trânsito entre o universo digital e o físico, da conexão entre o sentido da visão e o do tato.

As representações captam parte das coisas, subtraem, reduzem ou abstraem aspectos. O universo digital tem em sua essência a representação, pautado na 
experiência visual. A atribuição de cor a cada um dos pixels que estruturam a informação é possível a partir da construção de uma lógica algébrica associada ao fenômeno da cor. A Colorimetria, como técnica e ciência, estuda diferentes maneiras de descrever, quantificar e simular a percepção do fenômeno de interação da luz com a superfície dos materiais: a cor. Este fenômeno é percebido pelo olho e interpretado pelo cérebro. Desta maneira, envolve aspectos físicos, psicofísicos, psicométricos e visuais.

Externamente o dispositivo traz para o tato a abstração de um modelo de distribuição espacial da cor, parte essencial do universo digital. A parte interna do cubo fica dedicada essencialmente ao sentido da visão, onde a cor é percebida em seus valores de tom, brilho e saturação, representada por um caleidoscópio. Representações estas imperceptíveis aos invisuais tal como as cores no universo digital. Na parte externa, a representação tátil da cor é imperceptível aos visuais, necessitando ser traduzida para atribuir sentido à álgebra das cores. Desta maneira, o dispositivo constrói uma narrativa que explicita uma contraposição entre as representações físicas e digitais, entre um sentido de proximidade, como se configura o tato, com um sentido de distância, como é a visão.

Este exercício, que compreende técnicas de desenho paramétrico propiciou o trânsito entre modelos tridimensionais e abstratos, a partir da construção de definições algébricas utilizando-se de programação visual.

O uso da fabricação digital para a produção do dispositivo se apresenta como veículo de diálogo entre estes dois sentidos, entre o universo físico e o digital, com o propósito de construção de um desenho universal. Nesta direção, o exercício permitiu repensar o tema "o sentido e universo digital" junto a este contexto de produção e considera-lo atualmente pertinente e com potencial para promover novas reflexões.

Como um dos desdobramentos deste trabalho foi construído um material didático envolvendo uma narrativa sobre os conceitos e as técnicas envolvidas.

As reflexões foram provocadas especialmente por leituras referentes à teoria da escalada da abstração de Vilém Flusser. Nessa escala abstracional, o primeiro nível é o tridimensional, mediado pelo corpo. O segundo nível é o bidimensional, estando no plano das imagens. O terceiro é o unidimensional, o texto descreve a imagem. $O$ ápice da abstração é o que Flusser denomina de nulodimensional, referindo-se às imagens técnicas que podem ser codificadas por números. Esta lógica induz à associação desta adimensionalidade à linguagem binária das representações digitais. $O$ modelo de cor luz é representado por esta linguagem. O sistema RGB, uma abstração da cor luz, especifica as proporções de vermelho, verde e azul, tendo o problema de não ser nada intuitivo, sendo difícil de imaginar uma cor mediante tais valores. Se configura 
como um cubo centrado na origem de coordenadas. Os tres eixos representam niveis de intensidades das referidas cores, e varíam entre 0 e 1. (Foley,1990)

\section{Materiais e Métodos}

A concepção da superfície externa do dispositivo Chromoscope esteve relacionada à tradução do modelo de distribuição espacial da cor, parte essencial do universo digital, em um objeto físico tridimensional. Utilizou-se o sistema RGB (Red, Green and Blue), um sistema aditivo de cor luz, no qual cada cor é definida pela quantidade de vermelho, verde e azul que a compõe, cujos valores podem variar de 0 , mínima intensidade, a 255, máxima intensidade (ROCHA, 2010). Neste sistema, todas as cores são identificadas por uma tripla ordenada (R; G; B), assim é possível associar cada uma das cores com pontos de coordenadas inteiras em um cubo com arestas de comprimento 255 (BRAZIL, 2008). Desta maneira, o sistema RGB pode ser representado a partir do modelo Color Cube, ilustrado pela imagem à esquerda da Figura 01, o qual representa a distribuição dos valores RGB sobre cada uma das faces do cubo. Nesta distribuição espacial, cada um dos oito vértices do cubo representa uma coordenada associada a uma cor: preto (mínima intensidade cromática 0,0,0), as cores primárias do sistema RGB (vermelho $(255,0,0)$, verde $(0,255,0)$ e azul $(0,0$, $255)$ ), as cores secundárias (amarelo $(255,255,0)$, magenta $(255,0,255)$ e ciano $(0$, $255,255)$ ) e a cor branca (máxima intensidade cromática 255, 255, 255), conforme a imagem à direita da Figura 01.

Figura 01: À esquerda, distribuição espacial do sistema RGB no modelo Color Cube; à direita representação das cores presentes nos vértices do modelo Color Cube.
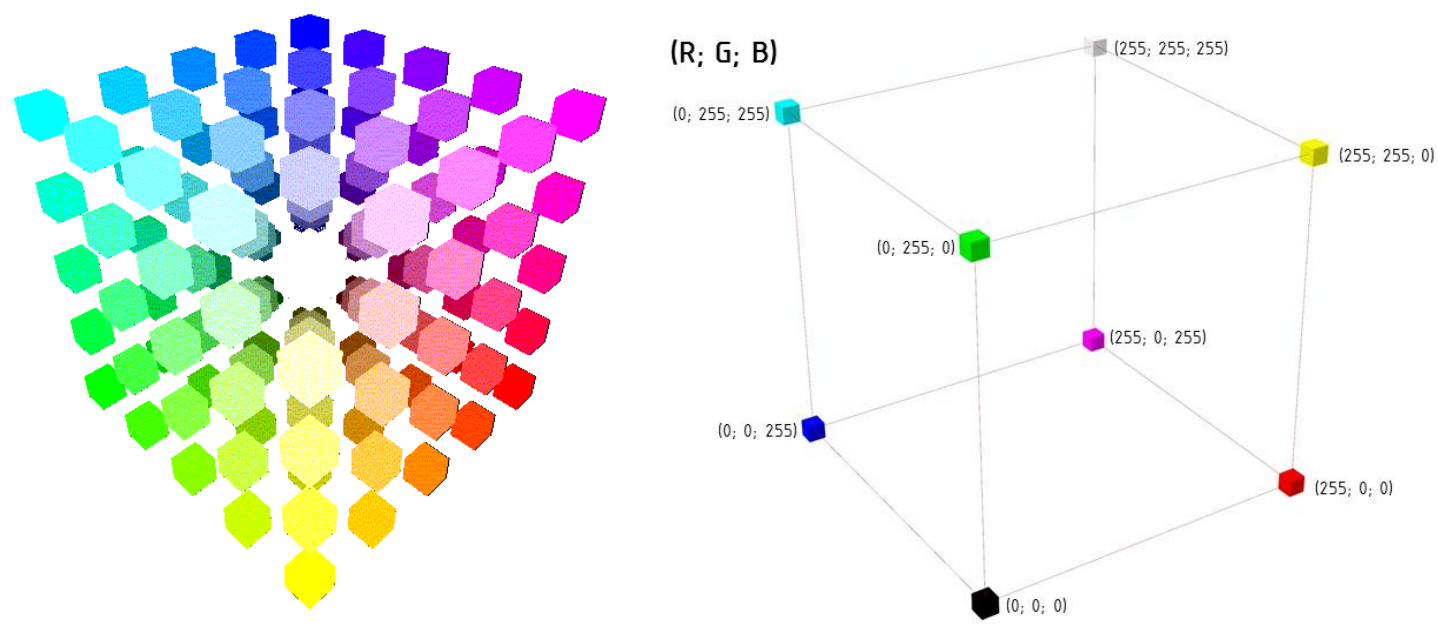

Fonte: colorcube.com/articles/basics/basics.htm (2000), autores (2016). 
A técnica utilizada para o composição da forma do dispositivo foi o desenho paramétrico, o qual se desenvolve em ambientes digitais e aplica algoritmos para a construção de definições. Algoritmos são entendidos como procedimentos para abordar problemas em um número finito de etapas, envolvendo a extração de princípios lógicos (TERZIDIS, 2006). O desenho paramétrico permite que as relações entre os elementos sejam explicitamente descritas, estabelecendo interdependências entre os objetos. A atribuição de diferentes valores aos parâmetros geram múltiplas variações, mantendo as condições topológicas da forma (OXMAN, 2006). Neste caso, para o uso do desenho paramétrico foram empregados o software Rhinoceros e o editor algorítmico Grasshopper, que combinados, permitem a geração de modelos associativos por meio de programação visual.

O modelo Color Cube, gerado a partir dos três inputs R (red), G (green) e B (blue), foi interpretado por meio de três formas geométricas associadas a estes valores: o cubo, correspondente à cor vermelha; o cone, à cor verde; e a esfera, à cor azul. O referencial utilizado para esta combinação entre formas e cores baseou-se em um postulado investigado por Wassily Kandinsky, um dos mestres da pintura da Bauhaus. De acordo com Gomez e Castral (2013), Kandisnky explora a relação entre cor, forma e significado, com o propósito de evocar sentimentos universais e elementares. A partir de seus estudos Kandinsky atribui o azul, cor fria e concencêtrica, à esfera; o vermelho, com sua força própria, ao cubo; e o amarelo, expansivo e superficial, ao triângulo (GOMEZ e CASTRAL, 2013), como ilustrado pela Figura 02. Para atender a demanda do dispositivo Chromoscope, a relação entre cor e forma foi adaptada, substituindo-se o amarelo pelo verde, e atribuindo-se tridimensionalidade às formas elementares, círculo/esfera, quadrado/cubo e triângulo/cone (Figura 03).

Figura 02: Relação entre cor e forma, segundo Kandinsky.

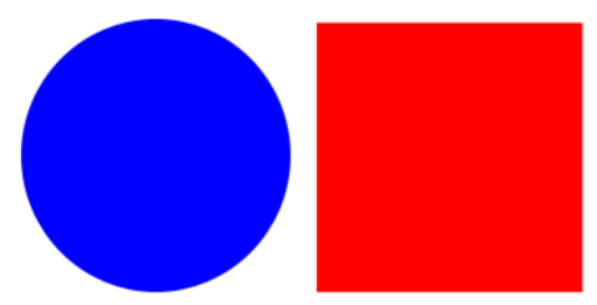

Fonte: Gomez e Castral (2013)

Figura 03: Atribuição de forma aos parâmetros RGB. 


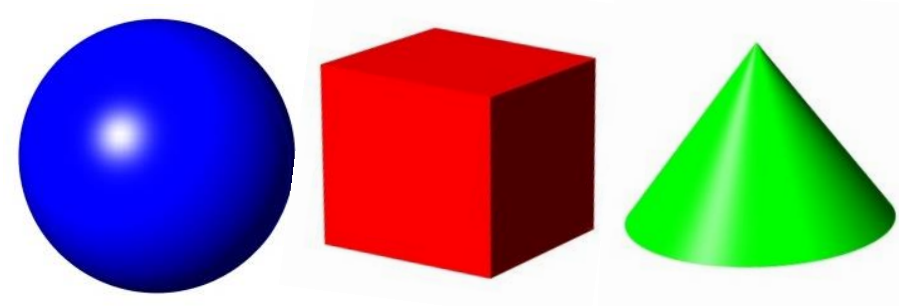

Fonte: Autores (2016)

\section{Desenvolvimento do dispositivo Chomoscope}

A configuração do Chromoscope partiu de um volume cúbico, tratando-se cada uma das seis faces como representações das lógicas de distribuição espacial da cor determinada pelo modelo Color Cube. Trabalhou-se, então, com interpolações algébricas, nas quais as intensidades das três cores atuaram como parâmetro para estabelecer o tamanho de cada uma das formas geométricas. Desta maneira, o tipo de forma e sua variação dimensional presente em cada face resultam das interpolações das três cores (formas) associadas a cada um de seus quatro vértices. Nas imagens superiores da Figura 02 pode-se observar esta lógica. À esquerda estão presentes as quatro cores que compõe os vértices desta face: ciano $(0,255,255)$, verde $(0,255,0)$, azul $(0,0,255)$ e preto $(0,0,0)$. Á direita a composição de formas que representa esta face. Nos cantos superior e inferior esquerdo a cor azul (esfera) está em seu valor máximo (255). Nos cantos da direita está em seu valor mínimo (0), tendo-se assim a ausência desta forma ao longo da aresta da direita desta face. A cor verde (cone) está em seu valor/dimensionamento máximo (255) nos vértices superiores, estando assim ausentes nos vértices inferiores (0). Enquanto que os quatro vértices estão caracterizados com valor zero para a cor vermelha, tendo-se a ausência total da forma cubo nesta face. Já na imagem inferior esquerda, do dispositivo em perspectiva, a face da direita ilustra a variação dimensional da cor vermelha (cubo), que auxilia então para compreender a ausência desta cor na face anteriormente apresentada.

Figura 04: Acima, composição de uma das faces do cubo; abaixo, modelo tridimensional completo e a programação visual que lhe deu origem. 

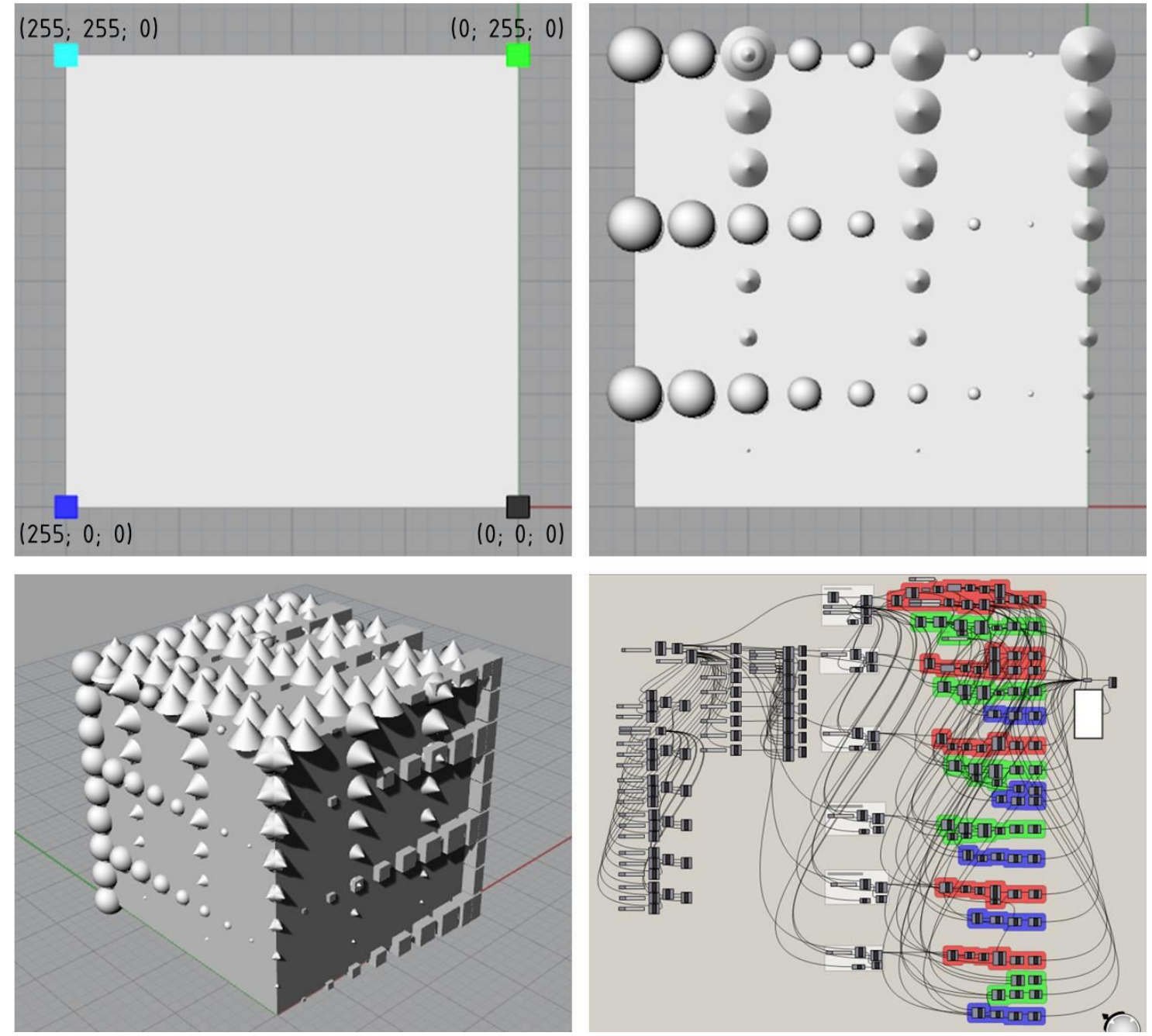

Fonte: Autores (2016)

A forma sofre ainda uma subtração para receber um tratamento diferenciado no seu interior através da presença de um caleidoscópio, conforme ilustrado na Figura 05. No vértice correspondente à posição da cor branca está a entrada de luz, enquanto que posição da cor preta está o espaço de visualização dos efeitos ópticos de simetrias por reflexão decorrentes do movimento de fragmentos da própria matéria em que foi fabricado o dispositivo: filamentos de PLA nas cores RGB. Representações estas imperceptíveis aos invisuais tal como as cores no universo digital. Externamente, a representação tátil da cor é imperceptível aos visuais, necessitando ser traduzida para atribuir sentido à álgebra das cores.

Figura 05: Caleidoscópio presente na parte interna do dispositivo e seus efeitos ópticos de simetrias por reflexão. 

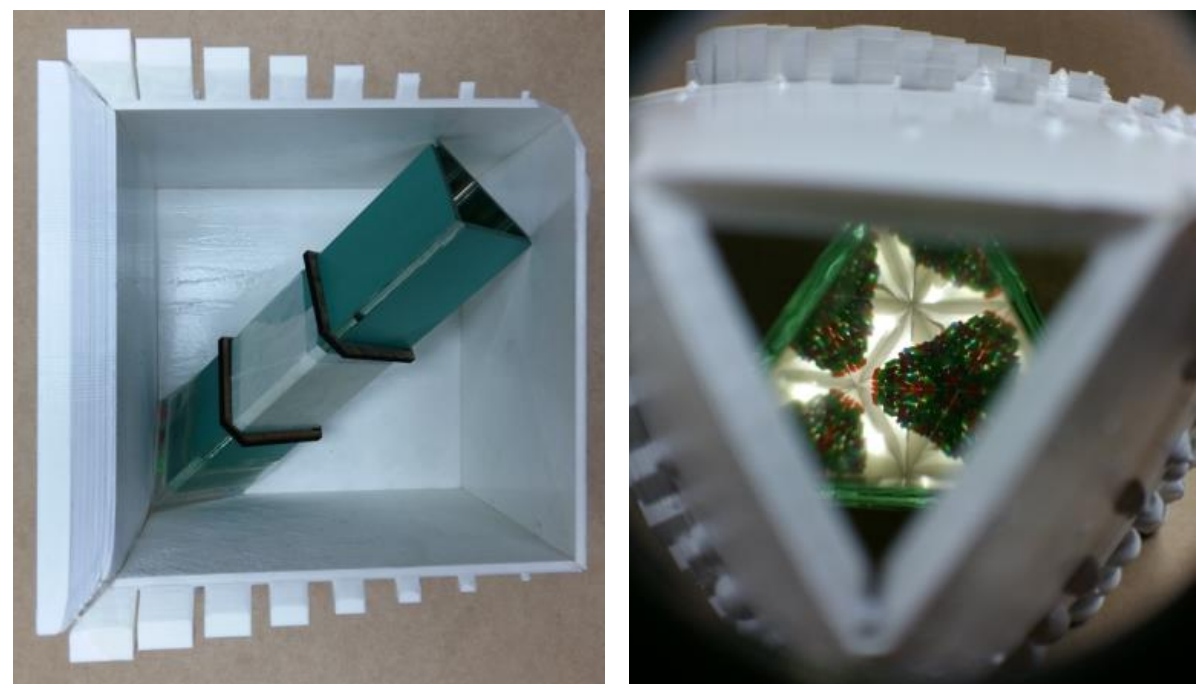

Fonte: Autores, 2016.

A prototipagem durante o processo de projeto e a fabricação do dispositivo foram relizadas por impressão 3D pela tecnologia FDM (Fused Deposition Modeling, em portugês, Modelagem por Fusão e Deposição) a partir de filamento de PLA (Poliácido Lático), material biodegradável, produzido a partir de fontes naturais. Assim como no processo de modelagem 3D, cada uma das faces foi impressa separadamente, conforme a Figura 06. Após a impressão de todas as faces, foram unidas e resultaram no dispositivo completo, ilustrado pela Figura 07.

Figura 06: Impressão 3D de uma das faces do dispositivo.

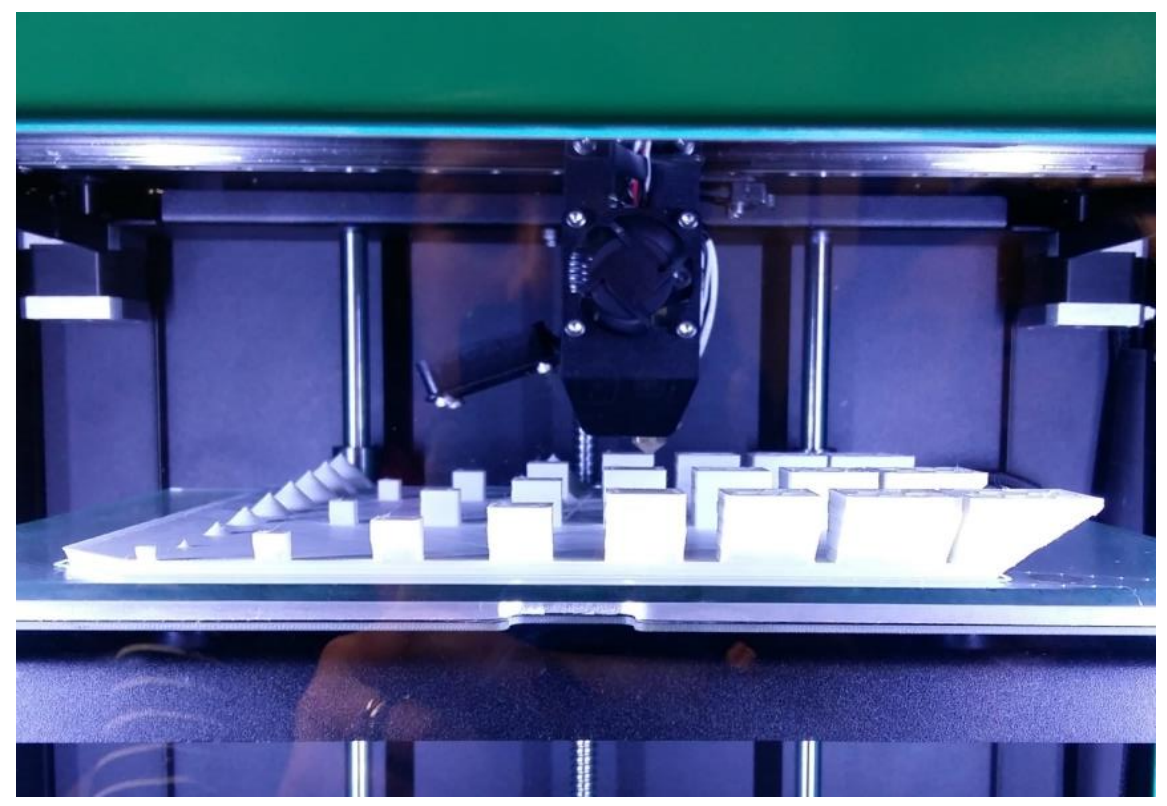

Fonte: Autores (2016)

Figura 07: Resultado final do dispositivo Chromoscope. 

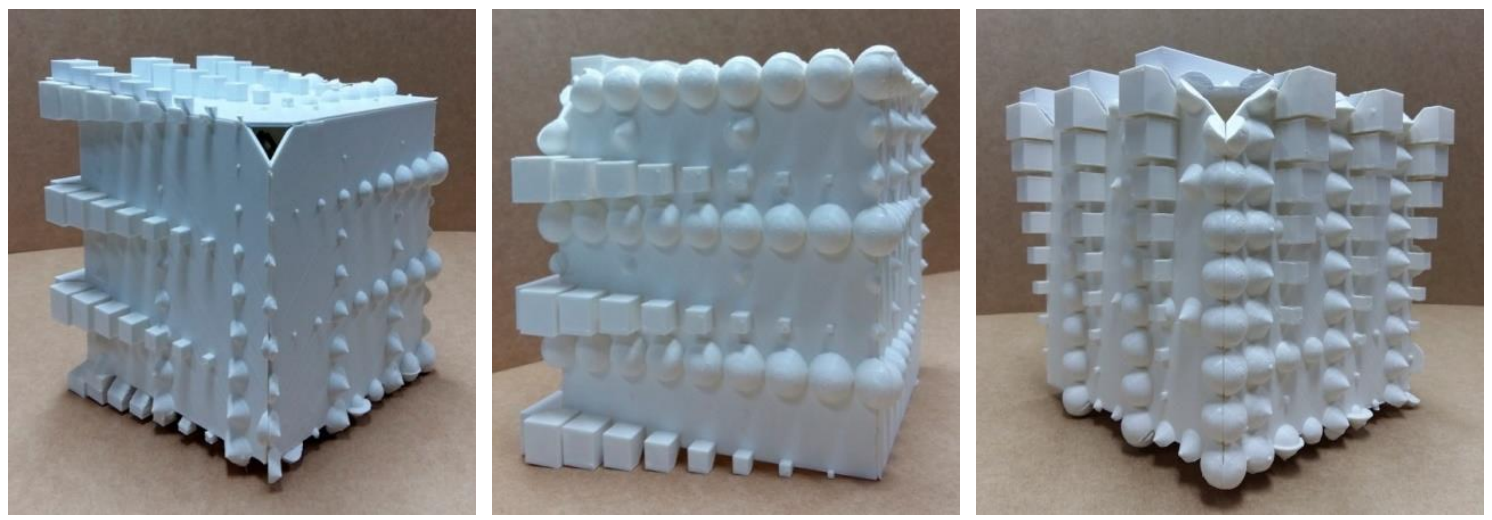

Fonte: Autores (2016)

\section{Considerações Finais}

O objeto Chromoscpe foi idealizado como um mediador - tátil/visual - que mostra através de seu processo uma experiência expandida dos sentidos. $O$ excesso de informação, é quase o "contrário da experiência", em meio ao bombardeio imagético na contemporaneidade, este trabalho é apresentado como um convite tátil ao sentido da visão. É um objeto constituído por camadas de significados que são resultado da articulação de dados e parâmetros, e não de uma escolha ou preferência formal, nesse processo os dados foram induzidos a visualidade que este objeto adquiriu.

Por outro lado, este trabalho é um dispositivo que necessita da ativação do outro, de alguém que o ative. Seu modo de existir, portanto, é enquanto proposição que convida o espectador a colocá-lo em prática, do contrário, torna-se um objeto inerte limitado ao olhar distante do observador. Ao olhar e sentir este objeto, o que vemos? O que sentimos? Nesse sentido, Chromoscope propõe a criação de um "olhar-tátil" para dentro de si, sobretudo, na expectativa de provocar modos possíveis de interação e reflexão sobre nossas sensações.

\section{Agradecimentos}

Ao CNPq e à UFPel, que tem apoiado o desenvolvimento do Projeto ACORDA (A....), subsidiando bolsas de iniciação científica e à SIGRADI (Sociedade Iberoamericana de Gráfica Digital) por ter promovido esta reflexão como parte da exposição ocorrida em 2016 junto ao Centro Cultural San Martín (Sarmiento, 1551, Buenos Aires) em que o Chromoscope foi exposto.

\section{Referências}

BRAZIL, André Luiz. Path Relinking and AES Cryptography in Color Image Steganography. Dissertação (Mestrado em Computação). Universidade Federal Fluminense, Niterói, 2008. 
FOLEY, James, VAN DAM, Andries, FEINER, Steven, HUGHES, John.1990. Computer Graphics. Principles and Practice. $2^{\text {nd }}$ ed. Addison-Wesley.

GOMEZ, Renan Santos; CASTRAL, Paulo César. Cor e Forma: estudo e análise das estruturas perceptivas representadas no livro 'Noturnos', de Cássio Vasconcellos. In: X International Conference on Graphics Engineering of Arts and Design e XXI Simpósio Nacional de Geometria Descritiva e Desenho Técnico. Florianópolis, 2013.

OXMAN, Rivka. Theory and design in the first digital age. In: Design Studies 27. London: Elsevier, 2006.

ROCHA, João Carlos. Cor luz, cor pigmento e os sistemas RGB e CMYK. Revista Belas Artes, São Paulo, n. 3, mai/ago de 2010. Disponível em: < http://www.belasartes.br/revistabelasartes/downloads/artigos/3/cor-luz-cor-pigmento-eos-sistemas-rgb-e-cmy.pdf> Acesso em: 06 de abril de 2017.

TERZIDIS, Kostas. Algorithmic Architecture. Burlington: Elsevier, 2006.

DIDI-HUBERMAN, Georges. Ser Crânio: Lugar, contato, pensamento, escultura. Belo Horizonte, Ed. C/Arte, 2009. p.87.

BONDÍA, Jorge Larrosa. Notas sobre a experiência e o saber de experiência.

In: Revista Brasi-leira de Educação. v. 19, Campinas: Jan/Fev/Mar/Abr, p. 20-28, 2002.

FLUSSER, Vilém. O mundo codificado: por uma filosofia do design e da comunicação. São Paulo: ed. Cosac Naify, 2013, p.224.

HEINLEIN, Robert. E ele construiu uma casa torta. Rio de Janeiro: Ed. Nova Fronteira S/A, 1966. 